\title{
Absence of Classical Lumps in Constrained Systems
}

\author{
R. Weder
}

Princeton University, Departments of Physics and Mathematics, Princeton, New Jersey 08540, USA

\begin{abstract}
We prove the absence of classical lumps for a large class of constrained systems. In particular we prove that there is no classical lump in the $O(N)$ non linear $\sigma$-model in 2 dimensional space time.
\end{abstract}

\section{Introduction}

The possibility of describing particles by quantization of classical lumps ${ }^{1}$ (i.e. finite energy classical solutions to the field equations in Minkowski space having the property that they confine permanently some of their energy in a bounded region of space) is presently the subject of a great deal of investigation. Methods to quantize classical lumps have been extensively studied (see [1-3] for example). Paradoxically, much less is known about the existence of classical lumps.

In this paper we prove that there are no classical lumps in a large class of constrained systems. In particular we prove that there are no classical lumps in the $O(N)$ non linear $\sigma$-model in 2 dimensional space-time. This example is particularly interesting because it is believed to be closely related to the Yang-Mills equations in 4 dimensional space-time, and because the $O(N)$ (in the case $N=3$ ) non linear $\sigma$ model in 2 dimensional space-time is believed to be equivalent to the Sine-Gordon equation [4].

From the technical point of view our result has the interesting feature that the system is not required to be scale invariant and the trace of the energy-momentum tensor is not required to be equal to zero.

There is some discrepancy in the definition of classical lumps in the literature. Here we consider a definition that contains most of the definitions found in the literature as particular cases. We will always consider non-singular solutions.

Definition I.1. A classical lump is a finite energy solution to the field equations in Minkowski space having the property that there exist $\varepsilon$ and $R>0$ such that for

1 They are also called solitons, classical glueballs and extended objects in the literature 
some $t_{0} \geqq 0$

$$
E_{R}(t)=\int_{|\bar{x}| \leqq R} \theta_{00}(t, \bar{x}) d^{D-1} x \geqq \varepsilon \quad \forall t \geqq t_{0} .
$$

$\theta_{00}(t, \bar{x})$ is the energy density and $D$ is the dimension of space-time.

The property that characterises classical lumps among finite-energy solutions is the ability to permanently confine some amount of their energy in a bounded region of space. Solutions describing a lump of energy traveling in space can be made to fit our definition by a change of reference frame.

We consider a system described by $N$ real fields $\phi_{a}(x), 1 \leqq a \leqq N$, in $D$ spacetime dimensions. The fields are required to satisfy the constrains:

$F_{b}(\phi)=0, \quad 1 \leqq b \leqq M$.

We denote $\phi=\left(\phi_{1}, \ldots, \phi_{N}\right)$.

The Lagrangian density is given by

$$
=1 / 2 g^{\mu v} \partial_{\mu} \phi_{a} \partial_{\nu} \phi_{a}-V(\bar{\phi})-\lambda_{b}(x) F_{b}(\bar{\phi}) \text {. }
$$

The $\lambda_{b}(x)$ are Lagrange multipliers. From the Euler-Lagrange equations we derive the field equations and the constrains:

$$
\begin{aligned}
\square \phi^{a} & =-\frac{\partial V}{\partial \phi_{a}}-\lambda_{b} \frac{\partial F_{b}}{\partial \phi_{a}}, \quad 1 \leqq a \leqq N, \\
F_{b}(\bar{\phi}) & =0, \quad 1 \leqq b \leqq M .
\end{aligned}
$$

It will be convenient to study the properties of the system under a scale transformation $\phi_{i}(x) \rightarrow \lambda^{d} \phi_{i}(\lambda x)$. Where $d$, a real number, is the scale dimension of the fields. The current associated with scale transformations is given by

$$
J^{\mu}=\partial^{\mu} \phi_{a}\left(d+x^{\nu} \partial_{\nu}\right) \phi_{a}-x^{\mu} \mathscr{L} .
$$

With the choice $d=\frac{D-2}{2}$ the free system is scale invariant, the scale current is conserved and the scale charge, $Q$, is time independent:

$$
Q=\int J^{0}(t, x) d^{D-1} x .
$$

We will not assume that the interacting system is scale invariant. Then the scale charge will not, in general, be time independent. In fact it is even not well defined, but when suitable modified and properly regularized it will be proven to be uniformly bounded in time. The method of proof of our theorem will be to show that this uniform bound is violated by a classical lump. Our theorem is

Theorem II.1. Assume that

$$
\begin{aligned}
& V \geqq 0 ; \quad(D-2) \frac{\partial V}{\partial \phi_{a}} \phi_{a} \geqq 2 D V ; \\
& (D-2) \frac{\partial F_{b}}{\partial \phi_{a}} \phi_{a}=0,1 \leqq b \leqq M .
\end{aligned}
$$

If $D \geqq 3$ assume $\left|\phi_{b}\right|^{p} \leqq C V(\bar{\phi})$, for some $p: 2 \leqq p<\frac{2(D-1)}{(D-3)}$, and $C>0,1 \leqq a \leqq N$. 
Then for every finite energy solution of the system

$$
\begin{aligned}
\square \phi_{a} & =-\frac{\partial V}{\partial \phi_{a}}-\lambda_{b} \frac{\partial F_{b}}{\partial \phi_{a}}, \quad 1 \leqq a \leqq N, \\
F_{b}(\bar{\phi}) & =0, \quad 1 \leqq b \leqq M .
\end{aligned}
$$

We have

$$
\lim _{t \rightarrow \infty} \inf E_{R}(t)=0 \text { for every } R>0 .
$$

Then clearly the system has no classical lumps.

An interesting particular case is the $0(N)$ non linear $\sigma$-model in 2 dimensional space-time (see Example 1). Particular cases in 3 and 4 dimensional space-time which are not scale invariant are discussed in Examples 3 and 4.

Several results in the absence of classical lumps exist in the literature. The older, known as "Derrick's theorem" [5] (see also Hobart [6]) is an argument to prove absence of time independent solutions (see [2] Appendix 2 for a generalization to gauge fields). Notice that this argument can be applied only if the appropriate boundary conditions are satisfied. For example these conditions are violated by the Prasad-Sommerfield magnetic monopole and dyon $[7,8][$ the boundary term is equal to $\left.\frac{4 \pi}{e^{2}}(\cosh \gamma-\sinh \gamma)(\lambda-1)\right]$ and it is for this reason that the Prasad-Sommerfield solutions $[7,8]$ are not excluded by the Hobart-Derrick argument. Other results have been obtained in the absence of time independent [2, 9], periodic [10], and non-radiant [11] solutions for the Yang-Mills equations. All these results require very strong assumptions in the behavior of the would-be solution at infinity.

In [12] we proved that the Yang-Mills equations have no classical lumps (in the sense of Definition I.1) without any assumption on the behavior of the wouldbe solution at infinity.

The method that $I$ invented in this paper has been applied in [14] to prove that after renormalization there are no instantons in four dimensional Euclidean space, i.e. that there are no finite action solutions to the Euler-Lagrange equations associated with the every effective lagrangian with an energy momentum tensor having the usual form of the trace anomaly [15].

\section{Constrained Systems}

We consider a system described by $N$ real fields $\phi_{a}(x), 1 \leqq a \leqq N$, where $x=\left(x_{0}, x_{1}, \ldots, x_{D-1}\right)$ belongs to $\mathbb{R}^{D}$, and $\phi_{a}(x)$ is a real valued function defined on $\mathbb{R}^{D}$. We will also use the notation $x_{0}=t, \bar{x}=\left(x_{1}, \ldots, x_{D-1}\right)$. We denote

$$
\bar{\phi}(x)=\left(\phi_{1}, \ldots, \phi_{N}\right) \text {. }
$$

The fields are required to satisfy the following constrains:

$$
F_{b}(\bar{\phi})=0, \quad 1 \leqq b \leqq M
$$


where the $F_{b}$ are functions from $\mathbb{R}^{N}$ to $\mathbb{R}$. The Lagrangian density of the system is given by

$$
\mathscr{L}=\frac{1}{2} g^{\mu v} \partial_{\mu} \phi_{a} \partial_{\nu} \phi_{a}-V(\bar{\phi})-\lambda_{b} F_{b}(\bar{\phi}) .
$$

The $\lambda_{b}(x)$ are Lagrange multipliers, the indices $\mu, v$ go from zero to $D-1$ and the usual convention of summation over repeated indices is assumed. The metric tensor $g_{\mu \nu}$ is diagonal and the diagonal components are $(1,-1,-1, \ldots,-1) . V(\bar{\phi})$, the potential, is a function from $\mathbb{R}^{N}$ to $\mathbb{R}$, and we denote $\partial_{\mu} \phi_{a}=\frac{\partial \phi_{a}}{\partial x_{\mu}}$.

From the Euler-Lagrange equations we obtain the evolution equations and the constrains.

$$
\begin{aligned}
& \square \phi_{a}=-\frac{\partial V}{\partial \phi_{a}}-\lambda_{b} \frac{\partial F_{b}}{\partial \phi_{a}}, \quad 1 \leqq a \leqq N, \\
& F_{b}(\bar{\phi})=0, \quad 1 \leqq b \leqq M,
\end{aligned}
$$

where $\square=\partial^{\mu} \partial_{\mu}$ is the D'Alambertian operator.

The energy-momentum tensor

$$
\theta_{\mu \nu}=\partial_{\mu} \phi_{a} \partial_{v} \phi_{a}-g_{\mu v} \mathscr{L}
$$

is conserved

$$
\partial_{\mu} \theta^{\mu \nu}=0
$$

and the energy integral, $E$, is independent of time

$$
E=\int \theta_{00}(t, \bar{x}) d^{D-1} x .
$$

It will be convenient to study the properties of our system under scale transformations. For this purpose we introduce the notion of scale invariance (here we follow [13]). Let $\phi_{a}(x), 1 \leqq a \leqq N$, be a set of $N$ real fields with Lagrangian density:

$$
\mathscr{L}=\mathscr{L}\left(\phi_{a}, \partial_{\mu} \phi_{a}\right)
$$

We define a scale transformation as follows

$$
\phi_{a}(x) \rightarrow \lambda^{d} \phi_{a}(\lambda x),
$$

where $d$, a real number, is the scale dimension of the fields. Under an infinitesimal transformation we have

$$
\delta \phi_{a}(x)=\left(d+x^{v} \partial_{v}\right) \phi_{a}(x) .
$$

The change in the Lagrangian density is

$$
\delta \mathscr{L}=\partial_{v}\left(x^{v} \mathscr{L}\right)-D \mathscr{L}+\pi_{a}^{\mu}(1+d) \partial_{\mu} \phi_{a}+d \frac{\partial \mathscr{L}}{\partial \phi_{a}} \phi_{a},
$$

where $\pi_{a}^{\mu}=\frac{\partial \mathscr{L}}{\partial \partial_{\mu} \phi_{a}}$. 


$$
\begin{aligned}
& \text { If } \\
& -D \mathscr{L}+\pi_{a}^{\mu}(1+d) \partial^{\mu} \phi_{a}+d \frac{\partial \mathscr{L}}{\partial \phi_{a}} \phi_{a} \equiv 0
\end{aligned}
$$

we have

$$
\delta \mathscr{L}=\partial_{v}\left(x^{v} \mathscr{L}\right)
$$

and the system is scale invariant. Using the Euler-Lagrange equations we obtain

$$
\partial \mu J^{\mu}=0
$$

where

$$
J^{\mu}=\pi_{a}^{\mu}\left(d+x^{\nu} \partial_{v}\right) \phi_{a}-x^{\mu} \mathscr{L}
$$

is the conserved current associated with scale invariance.

The canonical energy-momentum tensor is defined as follows

$$
\theta^{\mu v}=\pi_{a}^{\mu} \partial^{v} \phi_{a}-g^{\mu \nu} \mathscr{L} .
$$

The scale current can be written in terms of the energy-momentum tensor as follows

$$
J^{\mu}=\theta^{\mu v} x_{v}+d \pi_{a}^{\mu} \phi_{a} .
$$

We choose $d=\frac{D-2}{2}$, then

$$
J^{\mu}=\theta^{\mu v} x_{v}+\frac{D-2}{2} \pi_{a}^{\mu} \phi_{a}
$$

With this choice the free system is scale invariant and the free scale-charge, $Q$, is time independent

$$
Q=\int J_{0}(t, x) d^{D-1} x
$$

We will not assume that the interacting system is scale invariant. Then the scale current will not, in general, be conserved. In fact we have

$$
\partial_{\mu} J^{\mu}=\theta_{\mu}^{\mu}+\frac{D-2}{2} \partial_{\mu}\left(\phi_{a} J^{\mu} \phi_{a}\right)
$$

The scale charge will, in general, not be time independent. In fact it is even not well defined, but when suitably modified and properly regularized it will be proven to be uniformly bounded in time. The method of proof of our theorem is to show that this uniform bound is violated by a classical lump.

We denote by $E_{R}(t)$ the energy inside a sphere of radius $R$ at time $t$, i.e.

$$
E_{R}(t)=\int_{|\bar{x}| \leqq R} \theta_{00}(t, \bar{x}) d^{D-1} x
$$


Our main result is

Theorem II.1. Assume that $V \geqq 0 ; \quad(D-2) \frac{\partial V}{\partial \phi_{a}} \phi_{a} \geqq 2 D V ; \quad(D-2) \frac{\partial F_{b}}{\partial \phi_{a}} \phi_{a}=0$, $1 \leqq b \leqq M$. If $D \geqq 3$ assume $\left|\phi_{a}\right|^{p} \leqq C V(\bar{\phi})$, for some $p: 2 \leqq p<\frac{2(D-1)}{D-3}$, and $C>0$, $1 \leqq a \leqq N$.

Then for every finite energy solution of the system

$$
\begin{aligned}
& \square \phi_{a}=-\frac{\partial V}{\partial \phi_{a}}-\lambda_{b} \frac{\partial F_{b}}{\partial \phi_{a}}, \quad 1 \leqq a \leqq N, \\
& F_{b}(\bar{\phi})=0, \quad 1 \leqq b \leqq M .
\end{aligned}
$$

We have

$$
\lim _{t \rightarrow \infty} \inf E_{R}(t)=0, \text { for every } R>0 .
$$

Proof. It will be convenient to add counter-terms to the scale current. Instead of considering $J_{\mu}$ (II.19) we will study the current

$$
S^{\mu}=J^{\mu}-(D-2) \pi_{a}^{\mu} \phi_{a}-x_{0} \theta^{0 \mu} .
$$

The associated charge, $Q$, is defined as follows

$$
Q(t)=\int \omega(r) d r \int_{|\bar{x}| \leqq r} S^{0}(t, \bar{x}) d^{D-1} x .
$$

$\omega(r)$ is a bounded function of compact support. The cut-off $\omega(r)$ is introduced in order to make the charge $Q(t)$ well defined. We have

$$
\begin{gathered}
|Q(t)| \leqq E D N\|r \omega(r)\|_{1}+\frac{D-2}{2} \int \omega(r) d r \int_{|x| \leqq r}\left|\pi_{a}^{0} \phi_{a}\right| d^{D-1} x, \\
|Q(t)| \leqq E D N\|r \omega(r)\|_{1}+\frac{(D-2)}{\sqrt{2}} N C^{1 / p}\left[\frac{A}{D-1}\right]^{\frac{p-2}{2 p}} \\
\cdot E^{\frac{p+2}{2 p}}\left\|\frac{\frac{(D-1)(p-2)}{2 p}}{r} \omega(r)\right\|_{1} .
\end{gathered}
$$

Where $A$ is the area of the unit sphere in $\mathbb{R}^{D-1}$. This proves that the charge $Q(t)$ is uniformly bounded in time.

Now suppose that the thesis of the theorem is not true. Then there exist constants $\varepsilon, R, \geqq 0$ such that

$$
E_{R}(t) \geqq \varepsilon \quad \forall t \geqq t_{0} .
$$

We will prove that this violates the uniform bound (II.25).

By conservation of the energy momentum tensor

$$
\begin{aligned}
\partial_{t} Q(t)= & \int \omega(r) d r \int_{|x| \leqq r}\left[-\sum_{i, j=1}^{D-1} \partial_{j}\left(x^{i} \theta^{i j}\right)+\frac{D-2}{2} \sum_{j=1}^{D-1} \partial_{j}\left(\phi_{a} \partial^{j} \phi_{a}\right)+\theta^{00}\right. \\
& \left.+\frac{D-2}{2} \frac{\partial V}{\partial \phi_{a}} \phi^{a}-D V(\bar{\phi})\right] d^{D-1} x .
\end{aligned}
$$


Assume that support $\omega(r) \subset[R, \infty)$, then

$$
\partial_{t} Q(t) \geqq K,
$$

where

$$
\begin{aligned}
& K=\varepsilon\|\omega(r)\|_{1}-\left((D-1)^{2}+2\right) E\|r \omega(r)\|_{\infty}-\frac{D-2}{\sqrt{2}} N C^{1 / p} E^{\frac{p+2}{2 p}} . \\
& A^{\frac{p-2}{2 p}}\left\|\frac{(p-2)(D-2)}{2 p} \omega(r)\right\|_{\frac{2 p}{p-2}}
\end{aligned}
$$

We must choose a cut-off function $\omega(r)$ such that $K>0$. Take

$$
\omega(r)= \begin{cases}0 & r<R \\ \frac{1}{r} & R \leqq r \leqq R_{1} \\ 0 & r>R_{1} .\end{cases}
$$

Then if $R_{1}$ is large enough $K>0$ and

$$
Q(t) \geqq K t-Q(0),
$$

but (II.29) violates (II.25). Q.E.D.

We consider now several examples.

Example 1. Suppose $D=2, V(\bar{\phi}) \equiv 0$. Then for every finite energy solution of the constrained system

$$
\begin{aligned}
\square \phi^{a} & =-\lambda_{b} \frac{\partial F_{b}}{\partial \phi_{a}}, \quad 1 \leqq a \leqq N, \\
F_{b}(\bar{\phi}) & =0, \quad 1 \leqq b \leqq M .
\end{aligned}
$$

We have

$$
\lim _{t \rightarrow \infty} \inf E_{R}(t)=0, \text { for every } R>0 .
$$

If $M=1$ and $F_{1}(\bar{\phi})=\phi_{a} \phi_{a}-1$ this is the $0(N)$ non linear $\sigma$-model in one time and one space dimension.

Example 2. Suppose $D=3, \frac{\partial F_{b}}{\partial \phi_{a}} \phi_{a}=0,1 \leqq b \leqq M$, and $V(\bar{\phi})=\sum_{n=3}^{P} \lambda_{n}\left(\phi_{a} \phi_{a}\right)^{n}, \lambda_{i} \geqq 0$, and at least one of them strictly positive. Then Theorem II.1 applies and for every finite energy solution and every $R>0$

$$
\lim _{t \rightarrow \infty} \inf E_{R}(t)=0 .
$$

Example 3. Suppose $D=4, \frac{\partial F_{b}}{\partial \phi_{a}} \phi_{a}=0,1 \leqq b \leqq M$, and $V(\bar{\phi})=\sum_{n=2}^{P} \lambda_{n}\left(\phi_{a} \phi_{a}\right)^{n}, \lambda_{i}>0$ and $\lambda_{2}$ strictly positive. Then Theorem II.1 applies and for every finite energy solution and every $R>0$

$$
\lim _{t \rightarrow \infty} \inf E_{R}(t)=0 \text {. }
$$


Acknowledgements. I thank Prof. Louise Dolan for useful conversations in general aspects of gauge fields.

\section{References}

1. Daschen, R., Hasslacher, B., Neveu, A. : Phys. Rev. D10, 4114-4130 (1974); D11, 3424 (1975)

2. Coleman, S.: Lectures at the 1975 International School of Subnuclear Physics "Ettore Majorana"

3. Jackiw, R.: Quantum meaning of classical field theory. Rev. Mod. Phys. 49, 681-706 (1977)

4. Pohlmeyer, K.: Integrable Hamiltonian systems and interactions through quadratic constraints. Commun. math. Phys. 46, 207-221 (1976)

5. Derrick, G.H.: Comments on nonlinear wave equations as models for elementary particles. J. Math. Phys. 5, 1252-1254 (1964)

6. Hobart, R.H.: On the instability of a class of unitary field models. Proc. Phys. Soc. Lond. 82, 201 203 (1963)

7. Prasad, M.K., Sommerfield, C.M.: Phys. Rev. Letters 35, 760-762 (1975)

8. Bogomol'nyi, E. B.: The stability of classical solutions. Sov. J. Nucl. Phys. 24, 449-454 (1976)

9. Deser, S.: Absence of static solutions in source-free Yang-Mills theory. Phys. Lett. 64B, 463-464 (1976)

10. Pagels, H. : Absence of periodic solutions to scale invariant classical field theories. Phys. Lett. 68B, 466 (1977)

11. Coleman, S.: There are no classical glueballs. Commun. math. Phys. 55, 113-116 (1977)

12. Weder, R.: Absence of classical lumps. Commun. math. Phys. 57, 161-164 (1977)

13. Callan, C.G.,Jr., Coleman, S., Jackiw, R. : A new improved energy-momentum tensor. Ann. Phys. 59, 42-73 (1970)

14. Weder, R.: Instantons and renormalization. Preprint, Princeton University

15. Collins, J.C., Duncan, A., Joglekar, S. D.: Phys. Rev. D 16, 438 (1977)

Communicated by A. Jaffe

Received April 19, 1978 